\title{
Pathophysiology and Management of Long-term Complications After Transvaginal Urethral Diverticulectomy
}

\author{
Hyeon Woo Kim ${ }^{1,2}$, Jeong Zoo Lee ${ }^{1,2}$, Dong Gil Shin ${ }^{1,2}$ \\ ${ }^{1}$ Department of Urology, Pusan Nation University School of Medicine, Busan, Korea \\ ${ }^{2}$ Biomedical Research Institute, Pusan National University Hospital, Busan, Korea
}

Female urethral diverticulum (UD) is a rare and benign condition that presents as an epithelium-lined outpouching of the urethra. It has various symptoms, of which incontinence in the form of postmicturition dribble is the most common. The gold standard for the diagnosis of UD is magnetic resonance imaging, and the treatment of choice is transvaginal diverticulectomy. Despite the high success rate of transvaginal diverticulectomy, postoperative complications such as de novo stress urinary incontinence (SUI), recurrence, urethrovaginal fistula, recurrent urinary tract infections, newly-onset urgency, and urethral stricture can occur. De novo SUI is thought to result from weakening of the anatomical support of the urethra and bladder neck or damage to the urethral sphincter mechanism during diverticulectomy. It can be managed conservatively or may require surgical treatment such as a pubovaginal sling, Burch colposuspension, or urethral bulking agent injection. Concomitant SUI can be managed by concurrent or staged anti-incontinence surgery. Recurrent UD may be a newly formed diverticulum or the result of a remnant diverticulum from the previous diverticulectomy. In cases of recurrent UD requiring surgical repair, placing a rectus fascia pubovaginal sling may be an effective method to improve the surgical outcome. Urethrovaginal fistula is a rare, but devastating complication after urethral diverticulectomy; applying a Martius flap during fistula repair may improve the likelihood of a successful result. Malignancies in UD are rarely reported, and anterior pelvic exenteration is the recommended management in such cases.

Keywords: Urethral diseases; Diverticulum; Female; Postoperative complications

- Fund/Grant Support: This work was supported by Pusan National University Research Grant, 2020.

- Conflict of Interest: No potential conflict of interest relevant to this article was reported.

\section{INTRODUCTION}

Female urethral diverticulum (UD) presents as a localized epithelium-lined outpouching of the urethra located between the periurethral fibromuscular layer and anterior vaginal wall. Histopathologically, most UD cases are reported to have a benign nature, although some cases are identified as malignancies that require further management. Since the clinical presentation of $\mathrm{UD}$ is diverse and nonspecific, a high index of suspicion is the first step in the diagnostic evaluation to promptly treat the disease and avoid complications resulting from delayed management. Several conservative treatment methods such as endoscopic or open incision and drainage or marsupialization are available for UD, however, transvaginal surgical excision of UD has been described as the most definitive option for the disease in the literature. Despite the high success rate of urethral diverticulectomy, postoperative complications such as recurrence of $\mathrm{UD}$, de novo stress urinary incontinence (SUI), lower urinary

Corresponding author: Dong Gil Shin (ib https://orcid.org/0000-0002-9721-1706 Department of Urology, Pusan National University Hospital, 179 Gudeok-ro, Seo-gu, Busan 49241, Korea

Email: shindong16@hanmail.net

Submitted: January 1, 2021 / Accepted after revision: March 22, 2021 
tract symptoms (LUTS), recurrent urinary tract infections (UTIs), and urethrovaginal fistula are concerning problems for both clinicians and patients. The present review aims to summarize the postoperative complications following surgical diverticulectomy and their management.

\section{EPIDEMIOLOGY AND ETIOLOGY OF URETHRAL DIVERTICULUM}

Female UD is a rare disease, the prevalence of which ranges from $1 \%$ to $6 \%$ worldwide [1,2]. Although most UD patients present in their 30s and 40s, several studies have reported that the disease can be found in all age groups [3,4]. Since the symptoms of UD are nonspecific, it is often misdiagnosed as other conditions such as incontinence, recurrent UTI, and LUTS, delaying the proper management of the disease. UD is incidentally found in up to $1.4 \%$ of women who are evaluated for urinary incontinence and in more than $80 \%$ of patients who present with periurethral masses $[5,6]$.

The etiology of UD remains unclear; however, most cases are thought to be acquired rather than congenital. It is widely accepted that the development of UD begins from chronic obstruction of the periurethral glands, which causes dilatation of the gland lumen. When an abscess is formed due to repeated infection and inflammation of the obstructed gland, the abscess may rupture toward the urethral lumen with the formation of an ostium, which finally becomes a UD $[7,8]$. This process is demonstrated by the location of UD, which is consistent with that of periurethral glands, as well as the presence of fibrosis and inflammation in pathology examinations of excised UD specimens [9]. Obstetric trauma and iatrogenic injuries from urethral procedures, such as urinary incontinence surgery, are also possible etiologies for the development of UD [10-12].

\section{CLINICAL PRESENTATION AND DIAGNOSIS OF URETHRAL DIVERTICULUM}

The classic triad of presentation regarding UD is reported as the "3 D's": dysuria, dyspareunia, and a dribble of urine after micturition [13]. However, these 3 symptoms are not always present in many patients with UD, and co-occur in only about $20 \%$ of all cases [13]. The symptoms of most women with UD are vague, causing misdiagnosis; therefore, clinicians must have a high index of suspicion, especially in women with persistent LUTS without a definite corresponding disease [14]. The com- mon symptoms of UD include dysuria, dyspareunia, postmicturition dribble, frequency, urgency, localized pain, recurrent UTIs, anterior vaginal discomfort, vaginal mass, hematuria, and urethral discharge. In particular, incontinence in the form of postmicturition dribble is the most common symptom, and is found in $60 \%-70 \%$ of cases [15].

The diagnosis and evaluation of UD can be initiated from a physical examination with a high index of suspicion for the disease. A palpable periurethral mass is found in more than half of UD patients [14], and UD is diagnosed in over $80 \%$ of women with periurethral masses UD [6]. Once there is a high suspicion of UD based on the physical examination, the diagnosis can be confirmed by cystourethroscopy and imaging modalities such as balloon positive-pressure urethrography, voiding cystourethrography, computed tomography, ultrasound, or magnetic resonance imaging (MRI). These imaging studies are useful to evaluate the size, location, shape, and complexity of UD, and can be a blueprint for planning the direction of treatment. Currently, the gold standard for the diagnosis of UD is MRI, which provides invaluable information on the anatomic and tissue status of UD for planning a surgical procedure [15-17].

\section{TREATMENT OPTIONS}

Conservative management can be used in a limited number of UD patients who are asymptomatic and in whom malignancies can be excluded. Such patients can be managed by observation with close follow-up [18]. Other conservative management techniques such as postmicturition digital compression, prophylactic antibiotics, and needle aspiration can also be performed in women with minimally bothersome symptoms of UD [19].

The current treatment of choice for women with UD is transvaginal excision of the diverticulum, which has been reported to be the most effective management of the disease, with high success and low complication rates. Transvaginal excision of the diverticulum via an inverted $\mathrm{U}$ or midline incision on the anterior vaginal wall, followed by 3-layer closure with or without the interposition of a Martius fat pad or a vaginal flap, has been reported to have a success rate of $83 \%$ to $97 \%[12,20,21]$.

Transurethral diverticulotomy can be another option to manage UD; in this technique, the diverticular sac is opened into the urethra by using a diathermy electrode. This method may relieve UD-related symptoms by ensuring the decompression and drainage of the diverticular sac into the urethra [22,23]. 
However, this is not a definitive treatment method because the diverticular sac remains in place and UD can recur at any time after the procedure. Therefore, transurethral diverticulotomy is preferred in patients with diverticular abscesses for incision and drainage before undergoing delayed surgical diverticulectomy. Vaginal marsupialization of the diverticulum can be a treatment option for UD located in the distal portion of the urethra. Although the procedure may be simple and minimally invasive, it is only appropriate in distal UDs because the urethral sphincter is likely to be injured when the procedure is performed in UDs that are proximally located, and such an injury is highly likely to cause urinary incontinence [24].

\section{POSTOPERATIVE COMPLICATIONS OF TRANSVAGINAL URETHRAL DIVERTICULECTOMY}

Surgical excision of UD is an effective treatment option that has been reported to have a high success rate of up to $97 \%[12,20$, 21]. However, despite the high rate of success of transvaginal urethral diverticulectomy, there always exists a risk of potential postoperative complications that need to be reviewed with patients before treatment as part of the informed consent process. Complications that may occur specifically after urethral diverticulectomy include de novo SUI, recurrence of UD, urethro- vaginal fistula, recurrent UTIs, new-onset urgency, and urethral stricture [15]. Although these complications may not be common, they may substantially impact patients' quality of life. Therefore, both surgeons and patients should always be aware of these complications. The incidence and management of long-term complications after the surgical excision of UD are summarized in Table 1.

\section{De Novo SUI \\ Pathophysiology}

De novo SUI has been reported to occur in $1.7 \%$ to $33 \%$ of patients who undergo transvaginal urethral diverticulectomy $[13,25-34]$. Although de novo SUI is one of the most common complications after urethral diverticulectomy, its pathophysiology remains unclear. However, the necessity of extensive suburethral dissection in a large diverticulum and a proximal location of the diverticulum are thought to be factors compromising the anatomical support of the urethra and bladder neck or the damaging urethral sphincter mechanism, which may cause postoperative de novo SUI. Denervation injury during urethral diverticulectomy or damage of the urethral musculature and bladder neck by inflammation and the diverticulum itself may also cause SUI [32]. These theories are supported by several studies reporting that there tended to be an increased risk for the development of de novo SUI in proximally located and/or

Table 1. The incidence and management of long-term complications after the surgical excision of urethral diverticulum

\begin{tabular}{|c|c|c|}
\hline Postoperative complications & Incidence & Management \\
\hline \multirow{8}{*}{ De novo stress urinary incontinence } & $1.7 \%-33 \%$ & Conservative management \\
\hline & & 1. Physical therapy \\
\hline & & 2. Continence pessary \\
\hline & & Surgical management \\
\hline & & 1. Pubovaginal sling \\
\hline & & 2. Burch colposuspension \\
\hline & & 3. Urethral bulking agent injection \\
\hline & & 4. Tension-free midurethral sling \\
\hline \multirow[t]{2}{*}{ Recurrent urethral diverticulum } & $2 \%-16 \%$ & 1. Observation \\
\hline & & 2. Secondary surgical excision of urethral diverticulum \\
\hline \multirow[t]{2}{*}{ Urethrovaginal fistula } & $0.9 \%-8.3 \%$ & 1. Observation \\
\hline & & 2. Surgical repair of fistula \\
\hline \multirow[t]{3}{*}{ Urethral stricture } & $0 \%-5.2 \%$ & 1. Simple urethral dilatation \\
\hline & & 2. Simple meatotomy \\
\hline & & 3. Vaginal wall flap urethroplasty \\
\hline
\end{tabular}


large size $(>3 \mathrm{~cm})$ and/or circumferential-shaped diverticula according to MRI [31,32,35-38]. Therefore, patients who are continent preoperatively should always be warned about the possibility of SUI after urethral diverticulectomy [18].

\section{Treatment}

Most cases of postoperative de novo SUI improve with time and are usually conservatively managed with physical therapy or continence pessary. However, up to $29 \%$ of the patients may require surgical treatment such as a pubovaginal sling, Burch colposuspension, or urethral bulking agent injection. Tension-free vaginal tape midurethral slings have also been reported to be a treatment option that can be successfully and safely placed [32]. Since synthetic midurethral slings can potentially increase the risk of diverticular recurrence, mesh erosion, and urethrovaginal fistula, some studies have suggested that slings should be contraindicated for managing de novo SUI after urethral diverticulectomy [30,39]. Therefore, surgeons should always warn patients of the possible risks following the placement of synthetic midurethral slings, and patients should weigh the advantages (e.g., minimal invasiveness, fast recovery, and less postoperative pain and voiding dysfunction) and disadvantages (mesh-related complications associated with prior urethral diverticulectomy) of midurethral slings before selecting a management option for de novo SUI [18].

\section{Management of UD with concomitant SUI}

Concomitant urinary incontinence is a common symptom reported by $10 \%-57 \%$ of UD patients [13,14,25,30]. Although some patients may count postvoid dribbling caused by the emptying of the diverticulum as incontinence, studies have reported that true concomitant SUI is found in up to approximately $50 \%$ of UD patients $[25,40]$. Since pre-existing SUI may disappear after surgical diverticulectomy in $50 \%-100 \%$ of patients [41], a urodynamic study should be performed before urethral diverticulectomy to decide whether concomitant antiincontinence surgery is necessary to facilitate patient counseling regarding the treatment plan.

An important decision that should be made before urethral diverticulectomy in UD patients with SUI is whether to perform anti-incontinence surgery concurrently or to delay the procedure until the confirmation of persistent SUI postoperatively. Since SUI symptoms may disappear after urethral diverticulectomy in a certain number of patients, the decision of whether to perform concurrent anti-incontinence surgery is ambiguous [32]. Once a surgeon decides to perform concurrent anti-incontinence surgery during urethral diverticulectomy, placement of an autologous pubovaginal sling is a feasible and safe option that offers a high cure rate (up to $88 \%-100 \%$ ) for SUI $[14,42-44]$. The concurrent surgery may also have the advantage of preventing the need for a subsequent anti-incontinence procedure [16].

Bladder neck suspension has also been reported to be an effective procedure that could be performed during urethral diverticulectomy; according to Ganabathi et al. [25], 78\% of SUI cases were reported to be cured in UD patients who underwent concurrent diverticulectomy and bladder neck suspension. However, since an autologous pubovaginal sling may act as another tissue layer and support, which may reinforce the diverticulum repair, it may be preferable in cases of suspected intrinsic sphincter deficiency due to urethral sphincter disruption from the diverticulum itself or the surgical resection $[16,18]$.

Although sling placement at the time of diverticulectomy has shown a high cure rate of concomitant SUI, complications following concurrent surgery can also occur. Among patients who underwent urethral diverticulectomy with concurrent sling placement, wound infection was reported in $5 \%$, diverticulum recurrence in 5\%-14\%, de novo detrusor overactivity in 5\%$12 \%$, and transient urinary retention requiring temporary postoperative urethral catheterization in 3\%-62\% [42-44]. Since synthetic slings have the risk of infection, urethral erosion, and fistula formation during diverticulectomy, they are avoided during diverticulectomy and are even contraindicated if the urethra is entered during surgery [16].

The potential risk of complications regarding the concurrent anti-incontinence procedure and the minimal patient bother $[4,31]$ associated with mild SUI after urethral diverticulectomy have demonstrated that staged anti-incontinence surgery can be another feasible option for UD patients with concomitant SUI. Staged surgery can also avoid unnecessary anti-incontinence surgery for those who experience the disappearance of SUI symptoms after urethral diverticulectomy. In fact, a study in 2014 reported that concomitant SUI was resolved in $62 \%$ of UD patients after urethral diverticulectomy [4]. If concurrent SUI persists after diverticulectomy, secondary anti-incontinence procedures are usually performed about 6-8 months after the initial surgery $[4,31,32]$. Staged anti-incontinence surgery is required in approximately $4 \%-10 \%$ of patients after diverticulectomy $[31,32,34]$, and the success rate reaches up to $100 \%$, although $23 \%$ of these patients are reported to experi- 
ence urinary retention [4]. Staged anti-incontinence surgery may also be required in patients with de novo SUI after diverticulectomy, especially in those who are expected to have preoperative SUI that is masked by the mass effect of UD. Such de novo SUI has been reported to occur in approximately $10 \%-$ $33 \%$ of UD cases that are $>3 \mathrm{~cm}$ and/or proximally located $[4,29,31,32,34]$. Because concurrent SUI can regress after diverticulectomy and performing a concomitant anti-incontinence procedure increases the operative time, which may lead to increased postoperative morbidity, surgeons who prefer delayed anti-incontinence surgery should consider delaying the urodynamic study until the patient complains of persistent SUI even after diverticulectomy is successfully performed. Currently, there is no consensus on whether to perform concomitant or staged anti-incontinence surgery in women who undergo urethral diverticulectomy. Thus, the severity of symptoms, urodynamic findings, and the patient's goals should be taken into account before deciding whether to perform concomitant antiincontinence procedures with urethral diverticulectomy [16].

\section{Recurrence of UD}

The presence of UD after urethral diverticulectomy that is presumed to have been performed successfully may be a newly formed diverticulum or a result of recurrence. Studies have reported that UD had a recurrence rate of $2 \%-16 \%$ after urethral diverticulectomy, with a reoperation rate of $2 \%-13 \%[4,30$, $34,40,45]$. The recurrence of UD, both symptomatic and asymptomatic, can easily be diagnosed by using transverse MRI [46]. A study conducted in 2011 [47] showed that among cases of recurrent UD diagnosed by MRI, 33\% initially had a horseshoe-shaped diverticulum and $66 \%$ initially had a circumferential diverticulum. However, no recurrence after the excision of a simple diverticulum was reported using MRI in that study [47].

The recurrence of UD is thought to be caused by remnant diverticulum from an incomplete excision of the original UD, active infection of the diverticulectomy site, difficult dissection during diverticulectomy, inadequate or excessive suture line tension, residual dead space, or other technical factors [48]. Such causes likely reflect the complexity of UD, which has been identified to be one of the greatest risk factors of UD recurrence potentially requiring secondary surgical excision. UD is regarded as complex when it is proximally located, loculated, larger than $3 \mathrm{~cm}$ in diameter, associated with prior pelvic or vaginal surgery, or horseshoe-shaped or circumferentially enfolds the urethra on a transverse image, as well as if the case involves multiple diverticula [35,36]. These features of complex UD have all been reported to be factors associated with recurrence except the loculated shape [32,35]. During repeated urethral diverticulectomy, placing a rectus fascia pubovaginal sling may be effective to reduce the risk of further recurrence [49].

A study published in 2011 [34] reported that $10.7 \%$ of 122 patients who underwent urethral diverticulectomy at a single institution during 12 years showed recurrence requiring repeat surgical excision. In this study, a large proportion (69.2\%) of subjects with recurrence had undergone previous urethral or vaginal surgery, and proximal diverticula, multiple diverticula, and prior pelvic or vaginal surgery were identified as other risk factors for recurrence. According to a study in 2002 [29], a delayed diagnosis ( $>12$ months) of the primary diverticulum, a diameter larger than $4 \mathrm{~cm}$, incomplete excision of the diverticular wall, and lateral or horseshoe-shaped diverticula were reported as risk factors for UD recurrence, as well as other complications such as urethrovaginal fistula and de novo SUI.

\section{Urethrovaginal Fistula}

Urethrovaginal fistula is a devastating complication after urethral diverticulectomy; its incidence rate is reported to range from $0.9 \%$ to $8.3 \%$ [25]. This complication is thought to be correlated to infection of the excision site, overlapping suture lines during urethral diverticulectomy, tension on the repaired site caused by insufficient urethral tissue, insufficient flaps or grafts with poor vascularization, multiple previous urethral surgical procedures, and an inadequate quality of tissue applied on the repair site [16].

A urethral fistula may not require surgical repair if it is located at the distal portion of the urethra beyond the sphincteric mechanism, although it may cause splitting of the urinary stream or vaginal voiding. However, patients with a urethral fistula located proximally to the sphincter causing urinary incontinence may require surgical repair. Since sufficient wound healing of the previous diverticulectomy site and optimal status of tissue quality affects the success rate of fistula repair, the procedure is usually recommended to be attempted at least 3 months after urethral diverticulectomy [50]. Applying an adjuvant tissue flap, such as a Martius flap, during closure to aid the fistula repair site may prevent further fistula recurrence. A Martius flap may also be useful during urethral diverticulectomy for preventing urethrovaginal fistula by providing a well-vascularized additional tissue layer during closure of the diverticulectomy site. Furthermore, a meticulous surgical technique, good 
hemostasis, avoidance of contamination, preservation of a wellvascularized anterior vaginal wall flap, and a multilayered closure should be combined with a Martius flap to prevent urethrovaginal fistula after urethral diverticulectomy [51].

\section{Other Complications}

Other postoperative complications, such as urethral stricture, recurrent UTIs, dysuria, de novo urgency, and urethritis also have been reported. Urethral stricture has been reported to occur in $0 \%-5.2 \%$ of cases after urethral diverticulectomy [ 16 , $30,40]$, and can be managed by simple urethral dilatation or surgical procedures such as simple meatotomy or vaginal wall flap urethroplasty. Most postoperative recurrent UTIs and dysuria cases persisted since before urethral diverticulectomy rather than occurring de novo; recurrent UTIs and dysuria were persistent up to $23 \%$ and $26 \%$ of patients, respectively [32,35], De novo urethritis rarely appears after urethral diverticulectomy and it is usually difficult to cure by any medical means. This condition may contribute to the formation of a new UD [35]. Antibiotics and analgesics may alleviate postoperative UTIs, dysuria, and urethritis, and anticholinergic agents or beta- 3 agonists may be effective for de novo urgency.

\section{MALIGNANCY IN DIVERTICULA}

Underlying malignancy may be present in a rare subset of patients with UD. According to a study published in 2008 [52], 6\% of female patients with UD presented invasive carcinoma in the pathology report. The majority of malignancy cases were classified as adenocarcinoma, while 1 case was clear cell carcinoma. Glandular dysplasia, intestinal metaplasia, and villous adenoma were also reported in a few patients, which are correlated to an increased risk of subsequent adenocarcinoma. Squamous cell and transitional cell carcinomas can also be rarely found in patients reported to have diverticular malignancies [53].

Diverticular malignancies may present with symptoms such as hematuria, urethral bleeding, dysuria, UTIs, and urinary obstruction [53]. On imaging, diverticular carcinoma can manifest as an irregular filling defect of the diverticulum or a mass within the diverticular lumen [52]. However, since MRI can miss the presence of diverticular carcinoma, and benign granulation tissue within diverticular space can be misdiagnosed as diverticular carcinoma, imaging studies may have low accuracy in diagnosing carcinoma [54,55]. Therefore, preoperative imaging of the UD should not be considered as a definitive diagnos- tic method to predict the presence of diverticular malignancies, underscoring the importance of a pathologic study of the resected UD specimen.

A clear consensus regarding the management of urethral diverticular carcinoma does not yet exist due to its rarity and varied histology. Since there is a high risk of local recurrence and metastasis after only simple excision of UD that is revealed to be malignant, anterior pelvic exenteration with urinary diversion is usually recommended to manage diverticular carcinoma [52]. Adjuvant chemoradiation has also been suggested with anterior pelvic exenteration [53].

\section{CONCLUSION}

Complications following urethral diverticulectomy may be rare events; however, they greatly impact quality of life and require proper management. The most common postoperative complication is de novo SUI, which can be successfully managed by conservative treatment or surgical interventions such as a pubovaginal sling, Burch colposuspension, or urethral bulking agent injection. In contrast, concomitant SUI can be effectively managed by either concurrent or staged anti-incontinence surgery. The prevalence of recurrent UD depends on the location, size, and morphology of the original UD, and recurrence is thought to be caused by remnant UD, the condition of the surgical wound, and technical factors related to surgery. As management, placing a rectus fascia pubovaginal sling may be an effective method to reduce the risk of further recurrence of UD. Urethrovaginal fistula is a rare, but devastating complication after urethral diverticulectomy, and applying a Martius flap during fistula repair may improve the likelihood of a successful result. Urethral diverticular carcinoma is rarely reported after urethral diverticulectomy, and anterior pelvic exenteration with urinary diversion is usually recommended to manage the condition. Complications after urethral diverticulectomy have a substantial impact on patients' quality of life; therefore, patients should receive counseling with informed consent before surgery and proper management should be performed once a complication occurs.

\section{AUTHOR CONTRIBUTION STATEMENT}

- Conceptualization: HWK, JZL, DGS

- Formal analysis: $H W K$

- Funding acquisition: DGS 
- Methodology: HWK

- Project administration: DGS

- Visualization: $H W K$

- Writing-original draft: $H W K$

- Writing-review \& editing: $H W K, J Z L, D G S$

\section{ORCID}

$\begin{array}{ll}\text { Hyeon Woo Kim } & 0000-0002-6742-6087 \\ \text { Jeong Zoo Lee } & 0000-0001-9044-4067 \\ \text { Dong Gil Shin } & 0000-0002-9721-1706\end{array}$

\section{REFERENCES}

1. Davis BL, Robinson DG. Diverticula of the female urethra: assay of 120 cases. J Urol 1970;104:850-3.

2. Ma ${ }^{\circ}$ rtensson O, Duchek M. Translabial ultrasonography with pulsed colour-Doppler in the diagnosis of female urethral diverticula. Scand J Urol Nephrol 1994;28:101-4.

3. Burrows LJ, Howden NL, Meyn L, Weber AM. Surgical procedures for urethral diverticula in women in the United States, 1979-1997. Int Urogynecol J Pelvic Floor Dysfunct 2005;16:158-61.

4. Reeves FA, Inman RD, Chapple CR. Management of symptomatic urethral diverticula in women: a single-centre experience. Eur Urol 2014;66:164-72.

5. Aldridge CW Jr, Beaton JH, Nanzig RP. A review of office urethroscopy and cystometry. Am J Obstet Gynecol 1978;131:432-7.

6. Blaivas JG, Flisser AJ, Bleustein CB, Panagopoulos G. Periurethral masses: etiology and diagnosis in a large series of women. Obstet Gynecol 2004;103:842-7.

7. Tsivian M, Tsivian A, Shreiber L, Sidi AA, Koren R. Female urethral diverticulum: a pathological insight. Int Urogynecol J Pelvic Floor Dysfunct 2009;20:957-60.

8. Huffman JW. The detailed anatomy of the para-urethral ducts in the adult human female. Am J Obstet Gynecol 1948;55:86-101.

9. Leach GE, Bavendam TG. Female urethral diverticula. J Urol 1987; 30:407-15.

10. Kumar D, Kaufman MR, Dmochowski RR. Case reports: periurethral bulking agents and presumed urethral diverticula. Int Urogynecol J 2011;22:1039-43.

11. Clemens JQ, Bushman W. Urethral diverticulum following transurethral collagen injection. J Urol 2001;166:626.

12. Hammad FT. TVT can also cause urethral diverticulum. Int Urogynecol J Pelvic Floor Dysfunct 2007;18:467-9.

13. Ockrim JL, Allen DJ, Shah PJ, Greenwell TJ. A tertiary experience of urethral diverticulectomy: diagnosis, imaging and surgical outcomes. BJU Int 2009;103:1550-4.

14. Romanzi LJ, Groutz A, Blaivas JG. Urethral diverticulum in women: diverse presentations resulting in diagnostic delay and mismanagement. J Urol 2000;164:428-33.

15. Greenwell TJ, Spilotros M. Urethral diverticula in women. Nat Rev Urol 2015;12:671-80.

16. Crescenze IM, Goldman HB. Female urethral diverticulum: current diagnosis and management. Curr Urol Rep 2015;16:71.

17. Foster RT, Amundsen CL, Webster GD. The utility of magnetic resonance imaging for diagnosis and surgical planning before transvaginal periurethral diverticulectomy in women. Int Urogynecol J Pelvic Floor Dysfunct 2007;18:315-9.

18. Antosh DD, Gutman RE. Diagnosis and management of female urethral diverticulum. Female Pelvic Med Reconstr Surg 2011;17: 264-71.

19. Fortunato P, Schettini M, Gallucci M. Diagnosis and therapy of the female urethral diverticula. Int Urogynecol J Pelvic Floor Dysfunct 2001;12:51-7.

20. Tancer MI, Ravski NA. Suburethral diverticulae. Clin Obstet Gynaecol 1982;25:831-7.

21. Shalev M, Mistry S, Kernhan K, Miles BJ. Squamous cell carcinoma in a female urethral diverticulum. Urology 2002;59:773.

22. Lapides J. Transurethral treatment of urethral diverticula in women. J Urol 1979;121:736-8.

23. Satio $S$. Usefulness of diagnosis by the urethroscopy under anesthesia and effect of transurethral electrocoagulation in symptomatic female urethral diverticula. J Endourol 2000;14:455-7.

24. Spence HM, Duckett JW Jr. Diverticulum of the female urethra: clinical aspects and presentation of a simple operative technique for cure. J Urol 1970;104:432-7.

25. Ganabathi K, Leach GE, Zimmern PE, Dmochowski R. Experience with the management of urethral diverticulum in 63 women. J Urol 1994;152:1445-52.

26. Wharton LR Jr, Te Linde RW. Urethral diverticulum. Obstet Gynecol 1956;7:503-9.

27. Boatwright DC, Moore V. Suburethral diverticula in the female. J Urol 1963;89:581-4.

28. Ward JN. Technique to visualize urethral diverticula in female patients. Surg Gynecol Obstet 1989;168:278-9.

29. Porpiglia F, Destefanis P, Fiori C, Fontana D. Preoperative risk factors for surgery of female urethral diverticula. Urol Int 2002;69:711.

30. Ljungqvist L, Peeker R, Fall M. Female urethral diverticulum: 26year follow-up of a large series. J Urol 2007;177:219-24. 
31. Lee UJ, Goldman H, Moore C, Daneshgari F, Rackley RR, Vasavada SP. Rate of de novo stress urinary incontinence after urethal diverticulum repair. J Urol 2008;71:849-53.

32. Stav K, Dwyer PL, Rosamilia A, Chao F. Urinary symptoms before and after female urethral diverticulectomy-can we predict de novo stress urinary incontinence? J Urol 2008;180:2088-90.

33. Porten S, Kielb S. Diagnosis of female diverticula using magnetic resonance imaging. Adv Urol 2008;2008:213516.

34. Ingber MS, Firoozi F, Vasavada SP, Ching CB, Goldman HB, Moore CK, et al. Surgically corrected urethral diverticula: longterm voiding dysfunction and reoperation rates. J Urol 2011;77:65-9.

35. Ljungvist L, Peeker R, Fall M. Female urethral diverticulum26-year follow-up of a large series. J Urol 2007;177:219-24.

36. Malde S, Naaseri S, Kavia R, Pakzad M, Hamid R, Ockrim J, et al. Preliminary report on the effect of urethral diverticulum magnetic resonance imaging configuration on the incidence of new onset urodynamic stress urinary incontinence following excision. Urol Ann 2017;9:321-3.

37. Ginsberg P, Finkelstein LH. Urethral diverticulum with calculi: report of a case. J Am Osteopath Assoc 1983;82:588-90.

38. Juang CM, Horng HC, Yu HC, Chen CY, Chang CM, Yu KJ, et al. Combined diverticulectomy and anti-incontinence surgery for patients with urethral diverticulum and stress urinary incontinence: is anti-incontinence surgery really necessary? Taiwan J Obstet Gynecol 2006;45:67-9.

39. Vasavada SP. Urethral diverticula. In: Walters MD, Karram MM, editors. Urogynecology and reconstructive pelvic surgery. 3rd ed. Philadelphia (PA): Mosby Elsevier; 2007. p. 461-71.

40. Nickles SW, Ikwuezunma G, Mac Lachlan L, El-Zawahry A, Rames R, Rovner E. Simple vs complex urethral diverticulum: presentation and outcomes. Urology 2014;84:1516-9.

41. Wilson A, Hussain M, Hamid R, Ockrim J, Shah J, Greenwell T. Female urethral diverticula: evaluation of voiding dysfunction before and after surgery [abstract 1554]. J Urol 2012;187(4S):e630.

42. Faerber GJ. Urethral diverticulectomy and pubovaginal sling for simultaneous treatment of urethral diverticulum and intrinsic sphincter deficiency. Tech Urol 1998;4:192-7.

43. Swierzewski SJ 3rd, McGuire EJ. Pubovaginal sling for treatment of female stress urinary incontinence complicated by urethral diverticulum. J Urol 1993;149:1012-4.

44. Enemchukwu E, Lai C, Reynolds WS, Kaufman M, Dmochowski R. Autologous pubovaginal sling for the treatment of concomitant female urethral diverticula and stress urinary incontinence. J Urol 2015;85:1300-3.

45. Han DH, Jeong YS, Choo MS, Lee KS. Outcomes of surgery of female urethral diverticula classified using magnetic resonance imaging. Eur Urol 2007;51:1664-70.

46. Inneh IA. The combined influence of sociodemographic, preoperative comorbid and intraoperative factors on longer length of stay after elective primary total knee arthroplasty. J Arthroplasty 2015; 30:1883-6.

47. Shim JS, Oh MM, Kang JI, Ahn TS, Moon DG, Lee JG. Calculus in a female urethral diverticulum. Int Neururol J 2011;15:55-7.

48. Aspera AM, Rackley RR, Vasavada SP. Contemporary evaluation and management of the female urethral diverticulum. Urol Clin North Am 2002;29:617-24.

49. Migliari R, Pistolesi D, D’Urso L, Muto G. Recurrent pseudodiverticula of female urethra: five-year experience. Urology 2009;73: 1218-22.

50. Greiman AK, Rolef J, Rovner ES. Urethral diverticulum: a systematic review. Arab J Urol 2019;17:49-57.

51. Patel AK, Chapple CR. Female urethral diverticula. Curr Opin Urol 2006;16:248-54.

52. Thomas AA, Rackley RR, Lee U, Goldman HB, Vasavada SP, Hansel DE. Urethral diverticula in 90 female patients: a study with emphasis on neoplastic alterations. J Urol 2008;180:2463-7.

53. Ahmed K, Dasgupta R, Vats A, Nagpal K, Ashrafian H, Kaj B, et al. Urethral diverticular carcinoma: an overview of current trends in diagnosis and management. Int Urol Nephrol 2010;42:331-41.

54. Kim B, Hricak H, Tanagho EA. Diagnosis of urethral diverticula in women: value of MR imaging. AJR Am J Roentgenol 1993;161: 809-15.

55. Chung DE, Purohit RS, Girshman J, Blaivas JG. Urethral diverticula in women: discrepancies between magnetic resonance imaging and surgical findings. J Urol 2010;183:2265-9. 